\title{
ON SOME FREE BOUNDARY PROBLEMS FOR NAVIER-STOKES EQUATIONS
}

\author{
EWA ZADRZYŃSKA \\ Faculty of Mathematics and Information Sciences \\ Warsaw University of Technology \\ Pl. Politechniki 1, 00-661 Warszawa, Poland \\ E-mail: emzad@impan.gov.pl
}

\begin{abstract}
In this survey we report on existence results for some free boundary problems for equations describing motions of both incompressible and compressible viscous fluids. We also present ways of controlling free boundaries in two cases: a) when the free boundary is governed by surface tension, b) when surface tension does not occur.
\end{abstract}

1. Introduction. We present some results concerning free boundary problems for nonstationary Navier-Stokes equations. We are interested in a free boundary problem for equations describing the motion of a viscous general (heat-conducting) fluid which can be formulated as follows: find a domain $\Omega_{t} \subset \mathbb{R}^{3}$ with a boundary $S_{t}=S_{1} \cup S_{2 t}\left(S_{1}\right.$ is a fixed part of $S_{t}$ independent of time $t$; $S_{2 t}$ is a free part of $S_{t}$ ) as well as a velocity vector field $v=v(x, t)\left(v=\left(v_{1}, v_{2}, v_{3}\right)\right)$, density $\rho=\rho(x, t)$ and temperature $\theta=\theta(x, t)$ satisfying for $x \in \Omega_{t}, t \in(0, T), T>0$, the compressible Navier-Stokes system with initial conditions: $\left.\Omega_{t}\right|_{t=0}=\Omega,\left.v\right|_{t=0}=v_{0},\left.\rho\right|_{t=0}=\rho_{0},\left.\theta\right|_{t=0}=\theta_{0}$ in $\Omega$, the Dirichlet boundary condition for $v$ and $\theta$ on $S_{1} \times(0, T)$ and the Neumann type condition for the stress tensor and for $\theta$ on $\bigcup_{t \in(0, T)} S_{2 t} \times\{t\}$.

Therefore, the equations under consideration are as follows (see [LanLif], [Ser]):

$$
\begin{aligned}
& \rho\left[v_{t}+(v \cdot \nabla) v\right]-\operatorname{div} \mathbb{T}(v, p)=\rho \widetilde{f} \quad \text { in } \widetilde{\Omega}^{T}, \\
& \rho_{t}+\operatorname{div}(\rho v)=0 \quad \text { in } \widetilde{\Omega}^{T}, \\
& \rho c_{v}\left(\theta_{t}+v \cdot \nabla \theta\right)-\operatorname{div}(\varkappa \nabla \theta)+\theta p_{\theta} \operatorname{div} v \\
& -\frac{\mu}{2} \sum_{i, j=1}^{3}\left(v_{i x_{j}}+v_{j x_{i}}\right)^{2}-(\nu-\mu)(\operatorname{div} v)^{2}=\rho r, \quad \text { in } \widetilde{\Omega}^{T},
\end{aligned}
$$

2000 Mathematics Subject Classification: 35Q30, 35R35, 76D03, 76D05, 76N10.

Key words and phrases: Navier-Stokes equations, free boundary, incompressible viscous fluid, compressible viscous fluid, local existence, global existence, stability.

The paper is in final form and no version of it will be published elsewhere. 
where $T>0, \widetilde{\Omega}^{T} \equiv \bigcup_{t \in(0, T)} \Omega_{t} \times\{t\}, \Omega_{t} \subset \mathbb{R}^{3}$ is an unknown domain at time $t$ with boundary $S_{t}=S_{1} \cup S_{2 t} ; \mathbb{T}=\mathbb{T}(v, p)$ is the stress tensor given by

$$
\mathbb{T}(v, p)=\left\{-p \delta_{i j}+\mu\left(v_{i x_{j}}+v_{j x_{i}}\right)+(\nu-\mu) \operatorname{div} v \delta_{i j}\right\}_{i, j=1,2,3} .
$$

Moreover, $\widetilde{f}=\widetilde{f}(x, t)$ is the force per unit mass acting on the fluid; $r=r(x, t)$ denotes the heat sources per unit mass; $p=p(\rho, \theta)$ is the pressure, $\varkappa=\varkappa(\rho, \theta)$ the coefficient of heat conductivity, $c_{v}=c_{v}(\rho, \theta)$ the specific heat at constant volume; $\nu=\nu(\rho, \theta)$ and $\mu=\mu(\rho, \theta)$ are the viscosity coefficients. The functions $\varkappa, c_{v}, \nu, \mu$ are positive and $\nu>(1 / 3) \mu$.

We complete equations (1.1)-(1.3) with the following initial conditions:

$$
\begin{array}{ll}
\left.\Omega_{t}\right|_{t=0}=\Omega, & \left.S_{t}\right|_{t=0}=S, \\
\left.v\right|_{t=0}=v_{0} & \text { in } \Omega, \\
\left.\rho\right|_{t=0}=\rho_{0} & \text { in } \Omega, \\
\left.\theta\right|_{t=0}=\theta_{0} & \text { in } \Omega .
\end{array}
$$

We also complete system (1.1)-(1.3) with boundary conditions which depend on the geometry of $\Omega_{t}$. We consider two kinds of free boundary problems.

Drop problem. This is the problem describing the motion of an isolated mass of a fluid bounded by a free boundary. In this case $\Omega_{t} \subset \mathbb{R}^{3}$ is a bounded domain with the boundary $S_{t}=S_{2 t}\left(S_{1}=\emptyset\right)$.

For the drop problem the following boundary conditions are assumed:

$$
\begin{aligned}
& \mathbb{T} \bar{n}-\sigma H \bar{n}=-p_{0} \bar{n} \quad \text { on } \widetilde{S}^{T} \equiv \bigcup_{t \in(0, T)} S_{t} \times\{t\}, \\
& v \cdot \bar{n}=-\frac{\phi_{t}}{|\nabla \phi|} \quad \text { on } \widetilde{S}^{T}, \\
& \varkappa \frac{\partial \theta}{\partial n}=\bar{\theta} \quad \text { on } \widetilde{S}^{T}
\end{aligned}
$$

or

$$
\varkappa \frac{\partial \theta}{\partial n}=\varkappa_{a}\left(\theta_{a}-\theta\right) \quad \text { on } \widetilde{S}^{T}
$$

where $\bar{n}$ is the unit outward vector normal to $S_{t} ; \sigma$ is a constant coefficient of surface tension; $p_{0}$ is the constant external pressure; $\bar{\theta}=\bar{\theta}(x, t)$ the heat flow per unit surface; $\varkappa_{a}$ the constant coefficient of outer heat conductivity; $\theta_{a}$ the constant atmospheric temperature; $\phi(x, t)=0$ describes the boundary $S_{t} ; H$ denotes the double mean curvature of $S_{t}$ expressed by

$$
H \bar{n}=\Delta_{S_{t}}(t) x,
$$

where $\Delta_{S_{t}}(t)$ is the Laplace-Beltrami operator on $S_{t}$.

We can distinguish the two cases $\sigma>0$ and $\sigma=0$ in the boundary condition (1.8). If $\sigma>0$ we say that the free boundary is governed by surface tension.

Condition (1.9) is called the kinematic boundary condition. It means that fluid particles do not cross the free boundary. 
In drop problems it is usually assumed that

$$
\tilde{f}=f+k \nabla U
$$

where $f=f(x, t)$ denotes the external force field per unit mass; $k$ is the constant coefficient of gravitation, and $U(x, t)=\int_{\Omega_{t}} \frac{\rho(y, t)}{|x-y|} d y$ is the self-gravitational potential.

In the case of $k>0$ the second term on the right-hand side of (1.11) is called the self-gravitational force.

Surface waves problem. This is the problem describing the motion of a fluid occupying a semifinite domain in $\mathbb{R}^{3}$ bounded above by a free surface $S_{2 t} \equiv\left\{x=\left(x^{\prime}, x_{3}\right) \in \mathbb{R}^{3}\right.$ : $\left.x^{\prime} \in \mathbb{R}^{2}, x_{3}=F\left(x^{\prime}, t\right)\right\}$, and below by a fixed part of boundary $S_{t}$, that is, by $S_{1} \equiv\{x \in$ $\left.\mathbb{R}^{3}: x^{\prime} \in \mathbb{R}^{2}, x_{3}=-b\left(x^{\prime}\right)\right\}$, where $F$ is an unknown function and $b$ is a given function.

Therefore, initial condition (1.4) takes the form

$$
\left.F\right|_{t=0}=F_{0}\left(x^{\prime}\right), \quad x^{\prime} \in \mathbb{R}^{2} .
$$

For the surface waves problem the following boundary conditions are assumed:

$$
\begin{aligned}
& \mathbb{T} \bar{n}-\sigma H \bar{n}=-p_{0} \bar{n} \quad \text { on } \widetilde{S}_{2}^{T} \equiv \bigcup_{t \in(0, T)} S_{2 t} \times\{t\}, \\
& v \cdot \bar{n}=-\frac{F_{t}}{\sqrt{1+\left|\nabla_{x}^{\prime} F\right|^{2}}} \quad \text { on } \widetilde{S}_{2}^{T}, \\
& \varkappa \frac{\partial \theta}{\partial n}=\bar{\theta} \quad \text { on } \widetilde{S}_{2}^{T},
\end{aligned}
$$

or

$$
\begin{aligned}
& \varkappa \frac{\partial \theta}{\partial n}=\varkappa_{a}\left(\theta_{a}-\theta\right) \quad \text { on } \widetilde{S}_{2}^{T}, *\left(1.15^{\prime}\right) \\
& v=0 \quad \text { on } S_{1} \times(0, T), \\
& \theta=\theta_{b} \quad \text { on } S_{1} \times(0, T),
\end{aligned}
$$

where $\bar{n}$ is the unit outward vector normal to $S_{2 t}$; in (1.13) $\sigma>0$ or $\sigma=0$; $\theta_{b}$ is the constant temperature at $S_{1}, \nabla_{x}^{\prime}=\nabla_{x^{\prime}} ;(1.14)$ is the kinematic condition in this case.

Moreover, in surface waves problems it is usually assumed that

$$
\widetilde{f}=f-g e_{3},
$$

where $f=f(x, t)$ is an external force field per unit mass, $g$ denotes the accelaration of gravity and $e_{3}=^{t}(0,0,1)$.

Thus, the second term on the right-hand side of (1.18) is the gravity.

We can also consider some special cases of system (1.1)-(1.3).

$1^{o}$ Barotropic compressible fluid. This is a fluid of the state equation: $p=p(\rho)$. The free boundary problem for such a fluid is described by equations (1.1)-(1.2) (where the viscosity coefficients $\nu$ and $\mu$ are only functions of $\rho$ ) with conditions (1.4)-(1.6), (1.8)(1.9) or (1.5)-(1.6), (1.12)-(1.14), (1.16).

$2^{\circ}$ Incompressible fluid. Assuming that $\rho=$ const (let for simplicity $\rho=1$ ) equations (1.1)-(1.2) take the form of the classical Navier-Stokes equations

$$
v_{t}+(v \cdot \nabla) v-\nu \Delta v+\nabla p=\widetilde{f} \quad \text { in } \widetilde{\Omega}^{T},
$$




$$
\operatorname{div} v=0 \quad \text { in } \widetilde{\Omega}^{T},
$$

where $p=p(x, t)$.

The drop problem for an incompressible fluid takes the form of system (1.19)-(1.20) with initial conditions (1.4)-(1.5) and boundary conditions (1.8)-(1.9), where

$$
\mathbb{T}(v, p)=\left\{T_{i j}\right\}_{i, j=1,2,3}=\left\{-p \delta_{i j}+2 \nu\left(v_{i x_{j}}+v_{j x_{i}}\right)\right\}_{i, j=1,2,3} .
$$

The incompressible surface waves problem consists of equations (1.19)-(1.20) together with initial conditions (1.5), (1.12) and with boundary conditions (1.13)-(1.14), (1.16).

2. Existence results. Most of existence results are obtained after transforming a free boundary problem to a problem in a fixed domain. The most frequently used transformation connects the Eulerian coordinates $x$ with the Lagrangian coordinates $\xi$, which are defined as initial data for the following Cauchy problem:

$$
\frac{d x}{d t}=v(x, t), \quad x(0)=\xi, \quad \xi=\left(\xi_{1}, \xi_{2}, \xi_{3}\right) .
$$

Hence, the transformation connecting $x$ and $\xi$ coordinates has the form:

$$
x=\xi+\int_{0}^{t} u\left(\xi, t^{\prime}\right) d t^{\prime} \equiv X_{u}(\xi, t)
$$

where $u(\xi, t)=v\left(X_{u}(\xi, t), t\right)$.

In coordinates $\xi$ the above problems have the forms of problems with the unknown functions $u, \eta(\xi, t)=\rho\left(X_{u}(\xi, t), t\right), \vartheta(\xi, t)=\theta\left(X_{u}(\xi, t), t\right)\left(u\right.$ and $q(\xi, t)=p\left(X_{u}(\xi, t), t\right)$ in the incompressible case) in a fixed domain $\Omega^{T} \equiv \Omega \times(0, T)$. The drop problem for equations (1.1)-(1.3) in the Lagrangian coordinates takes the form:

$$
\begin{aligned}
& \eta u_{t}-\operatorname{div}_{u} \mathbb{T}_{u}(u, p)=\eta\left(g+k \nabla_{u} U_{u}\right) \quad \text { in } \Omega^{T}, \\
& \eta_{t}+\eta \nabla_{u} \cdot u=0 \quad \text { in } \Omega^{T}, \\
& \eta c_{v}(\eta, \vartheta) \vartheta_{t}-\nabla_{u} \cdot\left(\varkappa \nabla_{u} \vartheta\right)=-\vartheta p_{\vartheta}(\eta, \vartheta) \nabla_{u} \cdot u \\
& +\frac{\mu}{2} \sum_{i, j=1}^{3}\left(\xi_{x_{i}} \cdot \partial_{\xi} u_{j}+\xi_{x_{j}} \cdot \partial_{\xi} u_{i}\right)^{2}-(\nu-\mu)\left(\nabla_{u} \cdot u\right)^{2}=\eta h \quad \text { in } \Omega^{T}, \\
& \mathbb{T}_{u}(u, p) \bar{n}_{u}-\sigma \Delta_{u}(t) X_{u}=-q_{0} \bar{n}_{u} \quad \text { on } S^{T}, \\
& \varkappa(\eta, \vartheta) \bar{n}_{u} \cdot \nabla_{u} \vartheta=\bar{\vartheta} \quad \text { on } S^{T}
\end{aligned}
$$

or

$$
\begin{aligned}
& \varkappa(\eta, \vartheta) \bar{n}_{u} \cdot \nabla_{u} \vartheta=\varkappa_{a}\left(\vartheta_{a}-\vartheta\right) \quad \text { on } S^{T} \\
& \left.u\right|_{t=0}=v_{0},\left.\eta\right|_{t=0}=\rho_{0},\left.\vartheta\right|_{t=0}=\theta_{0}, \quad \text { in } \Omega,
\end{aligned}
$$

where $h(\xi, t)=r\left(X_{u}(\xi, t), t\right), \bar{\vartheta}(\xi, t)=\bar{\theta}\left(X_{u}(\xi, t), t\right)$,

$$
\begin{aligned}
\mathbb{T}_{u}(u, p)=\{ & -p(\eta, \vartheta) \delta_{i j}+\mu(\eta, \vartheta)\left(\partial_{x_{i}} \xi_{k} \partial_{\xi_{k}} u_{j}\right. \\
& \left.\left.+\partial_{x_{j}} \xi_{k} \partial_{\xi_{k}} u_{i}\right)+(\nu(\eta, \vartheta)-\mu(\eta, \vartheta)) \delta_{i j} \nabla_{u} \cdot u\right\}_{i, j=1,2,3},
\end{aligned}
$$

$\mathbf{I}=\left\{\delta_{i j}\right\}_{i, j=1,2,3}, \operatorname{div}_{u} \mathbb{T}_{u}(u, p)=\left\{\partial_{x_{j}} \xi_{k} \partial_{\xi_{k}} T_{u i j}(u, p)\right\}_{i=1,2,3} ;$ moreover $\nabla_{u}=\xi_{i x} \partial_{\xi_{i}}=$ $\left(\xi_{i x_{j}} \partial_{\xi_{i}}\right)_{j=1,2,3}, \xi_{i x_{j}}$ are the elements of the matrix $\xi_{x}$ which is inverse to $x_{\xi}=I+$ 
$\int_{0}^{t} u_{\xi}\left(\xi, t^{\prime}\right) d t^{\prime}=\left\{\delta_{i j}+\int_{0}^{t} u_{i \xi_{j}}\left(\xi, t^{\prime}\right) d t^{\prime}\right\}_{i, j=1,2,3}$ and the summation convention over repeated indices is assumed.

The surface waves problem can be transformed analogously.

The solvability results for the above free boundary problems are obtained in different function spaces. However, since the transformation (2.1) is involved in the nonlinear terms, we have always to impose such a regularity of solutions that

$$
\left\|\int_{0}^{T} u_{\xi} d t^{\prime}\right\|_{L_{\infty}(\Omega)} \leq T^{1 / 2}\left(\int_{0}^{T}\left\|u_{\xi}\right\|_{L_{\infty}(\Omega)}^{2} d t^{\prime}\right)^{1 / 2}<\infty .
$$

Therefore, for the presented free boundary problems we cannot expect the existence of solutions as weak as for initial-boundary value problems for the Navier-Stokes system in fixed domains. For this reason we can obtain for the above problems only local existence theorems or global existence theorems for initial data sufficiently close to equilibrium states.

Local existence and uniqueness. The local existence and uniqueness theorems for both the drop and surface waves problems have been proved in all cases, i.e. for incompressible, barotropic compressible and general compressible motions. The required regularity of function spaces in which we can examine the solvability of the above free boundary problems is implied by condition (2.8).

Below, we present the local solvability and uniqueness results in spaces of functions with the lowest regularity. First, we introduce the notation:

$$
\begin{aligned}
\mathcal{A}_{i T, \Omega_{i T}} \equiv & \mathcal{B}_{i T, \Omega_{i T}} \cap L_{2}\left(i T,(i+1) T ; W_{2}^{3}\left(\Omega_{i T}\right)\right), \\
\mathcal{B}_{i T, \Omega_{i T}} \equiv & \left\{w \in C\left([i T,(i+1) T] ; W_{2}^{2}\left(\Omega_{i T}\right)\right): w_{t} \in C\left([i T,(i+1) T] ; W_{2}^{1}\left(\Omega_{i T}\right)\right)\right. \\
& \cap L_{2}\left(i T,(i+1) T ; W_{2}^{2}\left(\Omega_{i T}\right)\right), w_{t t} \in C\left([i T,(i+1) T] ; L_{2}\left(\Omega_{i T}\right)\right) \\
& \left.\cap L_{2}\left(i T,(i+1) T ; W_{2}^{1}\left(\Omega_{i T}\right)\right)\right\} \quad \text { for } i \in \mathbb{N} \cup\{0\}
\end{aligned}
$$

and $\mathcal{A}_{0 T, \Omega_{0 T}} \equiv \mathcal{A}_{T, \Omega}, \mathcal{B}_{0 T, \Omega_{0 T}} \equiv \mathcal{B}_{0 T, \Omega}$.

Moreover, we denote by $W_{r}^{l, m}\left(\Omega^{T}\right)$, where $l, m \in \mathbb{R}_{+} \cup\{0\}, 1 \leq r<\infty$, the anisotropic Sobolev-Slobodetskiu space with the following norm

$$
\begin{aligned}
\|u\|_{W_{r}^{l, m}\left(\Omega^{T}\right)}^{r}= & \int_{\Omega^{T}}|u(x, t)|^{r} d x d t+\sum_{0<|\gamma| \leq[l]} \int_{\Omega^{T}}\left|D_{x}^{\gamma} u(x, t)\right|^{r} d x d t \\
& +\sum_{0<i \leq[m]} \int_{\Omega^{T}}\left|D_{t}^{i} u(x, t)\right|^{r} d x d t \\
& +\sum_{|\gamma|=[l]} \int_{0}^{T} d t \int_{\Omega} \int_{\Omega} \frac{\left|D_{x}^{\gamma} u(x, t)-D_{x^{\prime}}^{\gamma} u\left(x^{\prime}, t\right)\right|^{r}}{\left|x-x^{\prime}\right|^{n+r(l-[l])}} d x d x^{\prime} \\
& +\int_{\Omega} d x \int_{0}^{T} \int_{0}^{T} \frac{\left|D_{t}^{[m]} u(x, t)-D_{t^{\prime}}^{[m]} u\left(x, t^{\prime}\right)\right|^{r}}{\left|t-t^{\prime}\right|^{1+r(m-[m])}} d t d t^{\prime} .
\end{aligned}
$$

In (2.11) $D_{t}^{i}=\partial_{t}^{i}, D_{x}^{\gamma}=\partial_{x_{1}}^{\gamma_{1}} \ldots \partial_{x_{n}}^{\gamma_{n}}$ and $\gamma=\left(\gamma_{1}, \ldots, \gamma_{n}\right)$. In the case of integer $l$ the fourth term on the right-hand side of (2.4) is omitted and in the case of integer $m$ the fifth term is omitted. 
In our presentation of local existence results we assume for simplicity that $f=0$, $r=0, \bar{\theta}=0$ and we omit compatibility conditions. We can distinguish the following cases:

$1^{\circ}$ The incompressible case with $\sigma=0$ (drop problem with $k=0$ [Sol2], drop problem with $k>0$ [MZaj]; surface waves problem [A]).

Regularity of data: $r>3, v_{0} \in W_{r}^{2-2 / r}(\Omega), S \in W_{r}^{2-1 / r}$.

Regularity of solution: $u \in W_{r}^{2,1}\left(\Omega^{T}\right), q \in W_{r}^{1,0}\left(\Omega^{T}\right), q \in W_{2}^{1-1 / r, 1 / 2-1 /(2 r)}\left(S^{T}\right)$ in the case of the drop problem, and $q \in W_{2}^{1-1 / r, 1 / 2-1 /(2 r)}\left(S_{2} \times(0, T)\right)$ in the case of the surface waves problem (where $\left.S_{2} \equiv S_{2 t}\right|_{t=0}$ ), for some $T>0$.

$2^{\circ}$ The incompressible case with $\sigma>0$ (drop problem with $k=0$ [Sol1], drop problem with $k>0$ [Sol4]; surface waves problem [T]).

Regularity of data: $\alpha \in(1 / 2,1), v_{0} \in W_{2}^{1+\alpha}(\Omega), S \in W_{2}^{5 / 2+\alpha}$ for the drop problem, and $S_{1} \in W_{2}^{3 / 2+\alpha}, S_{2} \in W_{2}^{5 / 2+\alpha}$ for the surface waves problem.

Regularity of solution: $u \in W_{2}^{2+\alpha, 1+\alpha / 2}\left(\Omega^{T}\right), q \in W_{2}^{\alpha, \alpha / 2}\left(\Omega^{T}\right), \nabla q \in W_{2}^{\alpha, \alpha / 2}\left(\Omega^{T}\right)$ and $q \in W^{1 / 2+\alpha, 1 / 4+\alpha / 2}(S \times(0, T))$ for the drop problem, $q \in W^{1 / 2+\alpha, 1 / 4+\alpha / 2}\left(S_{2} \times(0, T)\right)$ in the case of the surface waves problem, for some $T>0$.

$3^{\circ}$ The compressible barotropic case with $\sigma>0$ (drop problem [SolT1]).

Regularity of data: $\alpha \in(1 / 2,1), v_{0} \in W_{2}^{1+\alpha}(\Omega), \rho_{0} \in W_{2}^{1+\alpha}(\Omega), S \in W_{2}^{5 / 2+\alpha}$, $p \in C^{3}\left(\mathbb{R}_{+}\right) ; \mu, \nu$ are constants.

Regularity of solution: $u \in W_{2}^{2+\alpha, 1+\alpha / 2}\left(\Omega^{T}\right), \eta \in W_{2}^{1+\alpha, 1 / 2+\alpha / 2}\left(\Omega^{T}\right)$ for some $T>0$.

$4^{\circ}$ The compressible heat-conducting case with $\sigma>0$ (drop problem with $k=0$ [Z1]).

Regularity of data: $\alpha \in[3 / 4,1), v_{0} \in W_{2}^{1+\alpha}(\Omega), \rho_{0} \in W_{2}^{1+\alpha}(\Omega), \theta_{0} \in W_{2}^{1+\alpha}(\Omega)$, $S \in W_{2}^{5 / 2+\alpha}, p \in C^{3}\left(\mathbb{R}^{2}\right), c_{v} \in C^{2}\left(\mathbb{R}^{2}\right), \nu \in C^{3}\left(\mathbb{R}^{2}\right), \mu \in C^{3}\left(\mathbb{R}^{2}\right), \varkappa \in C^{3}\left(\mathbb{R}^{2}\right)$.

Regularity of solution: $u \in W_{2}^{2+\alpha, 1+\alpha / 2}\left(\Omega^{T}\right), \vartheta \in W_{2}^{2+\alpha, 1+\alpha / 2}\left(\Omega^{T}\right)$, and $\eta \in$ $W_{2}^{1+\alpha, 1 / 2+\alpha / 2}\left(\Omega^{T}\right) \cap C\left([0, T] ; W_{2}^{1+\alpha}(\Omega)\right)$ for some $T>0$.

$5^{\circ}$ The compressible barotropic case with $\sigma=0$ (drop problem with $k=0$ [ZZaj1, ZZaj2]).

Regularity of data: $S \in W_{2}^{5 / 2}, v_{0} \in W_{2}^{2}(\Omega), \rho_{0} \in W_{2}^{2}(\Omega), u_{t}(0) \in W_{2}^{1}(\Omega), u_{t t}(0) \in$ $L_{2}(\Omega)$, (where $u_{t}(0), u_{t t}(0)$ are calculated from equation $\left.(2.2)\right), p \in C^{3}\left(\mathbb{R}^{2}\right) ; \mu, \nu$ are constants.

Regularity of solution: $u \in \mathcal{A}_{0 T, \Omega}, \eta \in \mathcal{B}_{0 T, \Omega}$ for some $T>0$.

$6^{o}$ The compressible barotropic case with $\sigma=0$ (drop problem with $k>0$ [StZaj]).

Regularity of data: $S \in W_{2}^{5 / 2}, v_{0} \in W_{2}^{2}(\Omega), \rho_{0} \in W_{2}^{2}(\Omega), p \in C^{3}\left(\mathbb{R}_{+}\right) ; \mu, \nu$ are constants.

Regularity of solution: $u \in L_{\infty}\left(0, T ; W_{2}^{1}(\Omega)\right) \cap L_{2}\left(0, T ; W_{2}^{3}(\Omega)\right), u_{t} \in L_{\infty}\left(0, T ; L_{2}(\Omega)\right)$ $\cap L_{2}\left(0, T ; W_{2}^{1}(\Omega)\right), \eta \in L_{\infty}\left(0, T ; W_{2}^{1}(\Omega)\right), \eta_{t} \in L_{\infty}\left(0, T ; L_{2}(\Omega)\right) \cap L_{2}\left(0, T ; H^{2}(\Omega)\right), \eta_{t t} \in$ $L_{2}\left(\Omega^{T}\right)$, for some $T>0$.

$7^{\circ}$ The compressible heat-conducting case with $\sigma=0$ (drop problem [ZZaj1]).

Regularity of data: $S \in W_{2}^{5 / 2}, v_{0} \in W_{2}^{2}(\Omega), \theta_{0} \in W_{2}^{2}(\Omega), \rho_{0} \in W_{2}^{2}(\Omega), u_{t}(0) \in$ $W_{2}^{1}(\Omega), \vartheta_{t}(0) \in W_{2}^{1}(\Omega), u_{t t}(0) \in L_{2}(\Omega), \vartheta_{t t}(0) \in L_{2}(\Omega)$ (where $u_{t}(0), u_{t t}(0), \vartheta_{t}(0)$, $\vartheta_{t t}(0)$ are calculated from equations $(2.2)$ and $\left.(2.4)\right), p \in C^{3}\left(\mathbb{R}^{2}\right), c_{v} \in C^{2}\left(\mathbb{R}^{2}\right) ; \mu, \nu, \varkappa$ are constants. 
Regularity of solution: $u \in \mathcal{A}_{0 T, \Omega}, \vartheta \in \mathcal{A}_{0 T, \Omega}, \eta \in \mathcal{B}_{0 T, \Omega}$ for some $T>0$. The full review of local existence results can be found in [Z2].

Global existence and stability. The global existence theorems for the three-dimensional free boundary problems can be proved for initial data close to equilibrium states. For incompressible motions it means that the initial velocity $v_{0}$ is assumed to be small. Moreover, for problems with a free boundary governed by surface tension it is assumed that a boundary of an initial domain is close to a sphere of radius $R_{0}=\left(\frac{3}{4 \pi}|\Omega|\right)^{1 / 3}$ in the case of drop problem or to a plane in the case of surface waves problem. Under the above assumptions it is proved that there exists a global solution which is close to the equilibrium state. It means that the velocity $v$ of the fluid remains small, the pressure is close to a certain constant and the free boundary $S_{t}$ remains close to the same sphere or to the same plane as the initial boundary $S$ for all $t>0$.

Solonnikov [Sol2] proved that in the case of the incompressible drop problem with $\sigma=0$ and $k=0$, the local solution (see $1^{\circ}$ above) can be extended to a global one if $v_{0}$ is sufficiently small in $W_{r}^{2-2 / r}(\Omega)$. For incompressible surface waves problem with $\sigma=0$ the global existence result with a small initial velocity has been obtained by Sylvester [Syl] in spaces of more regular functions.

In the incompressible case with $\sigma>0$ the local solutions described in $2^{\circ}$ above can be extended to global ones if $v_{0} \in W_{2}^{1+\alpha}(\Omega)$ and $S \in W_{2}^{5 / 2+\alpha}$ are sufficiently close to an equilibrium state (see [Sol1], [Sol3], [TTan]).

Similar results can be proved for compressible problems. In particular, an equilibrium state in the compressible heat-conducting case with $\sigma>0$ and $k=0$ can be defined as follows.

Definition 1. Let $f=0, r=\bar{\theta}=0$. By an equilibrium state we mean a solution $\left(v, \theta, \rho, \Omega_{t}\right)$ of problem (1.1)-(1.10) such that $v=0, \theta=\theta_{e}, \rho=\rho_{e}, \Omega_{t}=\Omega_{e}$ for $t \geq 0$, where $\rho_{e}=\left(M /(4 / 3) \pi R_{e}^{3}\right), M=\int_{\Omega} \rho_{0}(\xi) d \xi, \Omega_{e}$ is a ball of radius $R_{e} ; R_{e}>0$ and $\theta_{e}>0$ satisfy the equation

$$
p\left(\frac{M}{(4 / 3) \pi R_{e}^{2}}, \theta_{e}\right)=p_{0}+\frac{2 \sigma}{R_{e}} .
$$

In this case if we assume that $\alpha \in(3 / 4,1), v_{0} \in W_{2}^{1+\alpha}(\Omega)$ is sufficiently small, $\rho_{0} \in W_{2}^{1+\alpha}(\Omega), \theta_{0} \in W_{2}^{1+\alpha}(\Omega)$ are sufficiently close to $\rho_{e}$ and $\theta_{e}$, respectively, and the function $\widetilde{R}$ describing $S$ (see Section 3 ) is sufficiently close to $R_{e}$, then we can extend the local solution (described in $4^{\circ}$ above) to a global one (see [Z1], [Z2]).

The global solvability of the compressible barotropic drop problem with $\sigma>0$ is proved in [SolT2] in the function spaces determined by the local solution defined in $3^{\circ}$ and in [Zaj] in spaces of more regular functions. A global solvability result for the compressible barotropic surface waves problem with $\sigma>0$ can be found in [JinPad].

Moreover, Tani and Tanaka [TanT] proved the global existence theorem for the compressible heat-conducting surface waves problem with $\sigma>0$ in a class of functions of a greater regularity than the regularity described in $4^{\circ}$ for the drop problem.

Now, consider the case of $\sigma=0$ and $k=0$. For the compressible barotropic drop problem by an equilibrium state we mean a constant solution $\left(0, \rho_{e}, \Omega_{e}\right)$ of the problem 
with $f=0$ such that $p\left(\rho_{e}\right)=p_{0}$ and $\left|\Omega_{e}\right|=M / \rho_{e}$. In [ZZaj2] we prove that if initial data are close to the equilibrium solution then the local solution of the problem (see $5^{\circ}$ above) can be extended to a global one.

Introduce the notation:

$$
\begin{gathered}
p_{\sigma}=p-p_{0}, \quad \theta_{\sigma}=\theta-\theta_{e}, \quad \rho_{\sigma}=\rho-\rho_{e}, \\
\varphi(t)=|v(t)|_{2,0, \Omega_{t}}^{2}+\left|\theta_{\sigma}(t)\right|_{2,0, \Omega_{t}}^{2}+\left|\rho_{\sigma}(t)\right|_{2,0, \Omega_{t}}^{2}, \\
\Phi(t)=|v(t)|_{3,1, \Omega_{t}}^{2}+\left|\theta_{\sigma}(t)\right|_{3,1, \Omega_{t}}^{2}+\left\|\rho_{\sigma}(t)\right\|_{W_{2}^{2}\left(\Omega_{t}\right)}^{2}+ \\
+\left\|\rho_{\sigma t}(t)\right\|_{W_{2}^{2}\left(\Omega_{t}\right)}^{2}+\left\|\rho_{\sigma t t}(t)\right\|_{W_{2}^{1}\left(\Omega_{t}\right)}^{2}, \\
\mathfrak{M}(t)=\left\{\left(v, \theta_{\sigma}, \rho_{\sigma}\right): \sup _{0 \leq t^{\prime} \leq t} \varphi\left(t^{\prime}\right)+\int_{0}^{t} \Phi\left(t^{\prime}\right) d t^{\prime}<\infty\right\},
\end{gathered}
$$

where $|f(t)|_{l, k, \Omega_{t}} \equiv \sum_{i \leq l-k}\left\|\partial_{t}^{i} f(t)\right\|_{W_{2}^{l-i}\left(\Omega_{t}\right)}, l \in \mathbb{N} \cup\{0\}$.

From [ZZaj4] it follows that the local solution of problem (1.1)-(1.10) described in ro above can be extended to a global one if initial data are sufficiently close to the equlibrium state, i.e. $\varphi(0) \leq \varepsilon$ with $\varepsilon$ sufficiently small and if the following differential inequality holds:

$$
\frac{d \bar{\varphi}}{d t}+c_{1} \Phi \leq c_{2}\left[\varphi\left(1+\varphi^{2}\right)+\left\|\int_{0}^{t} v d t^{\prime}\right\|_{W_{2}^{3}\left(\Omega_{t}\right)}^{2}\right] \Phi \quad \text { for } t \leq T,
$$

where $c_{1}, c_{2}$ are positive constants depending on $\rho_{1}, \rho_{2}, \theta_{1}, \theta_{2}, \nu, \mu, \varkappa, c_{v}, p,\|S\|_{W_{2}^{5 / 2}}$ and the constants from the imbedding theorems and the Korn inequalities (being also nondecreasing continuous functions of $\left.\left\|\int_{0}^{t} v d t^{\prime}\right\|_{W_{2}^{3}\left(\Omega_{t}\right)}\right)$. Moreover, $\bar{\varphi}$ in (2.12) is a certain function satisfying the estimate

$$
c_{3} \varphi(t) \leq \bar{\varphi}(t) \leq c_{4} \varphi(t) \quad \text { for } t \leq T,
$$

where $c_{3}, c_{4}$ are positive constants depending on $\rho_{1}, \rho_{2}, \theta_{1}, \theta_{2}, \mu, \varkappa, c_{v}, p,\|S\|_{W_{2}^{5 / 2}}$ and the constants from the imbedding theorems.

In [ZZaj3] it is proved that inequality $(2.12)$ holds for problem $(1.1)-(1.9),\left(1.10^{\prime}\right)$. In this case we admit the following definition of an equilibrium state.

Definition 2. An equilibrium state is a solution $\left(v, \theta, \rho, \Omega_{t}\right)$ of $(1.1)-(1.9),\left(1.10^{\prime}\right)$ such that $v=0, \theta=\theta_{e}, \rho=\rho_{e}, \Omega_{t}=\Omega_{e}$ for $t \geq 0$, where $\theta_{e}=\theta_{a}, \rho_{e}$ is a positive constant satisfying the state equation

$$
p\left(\rho_{e}, \theta_{e}\right)=p_{0},
$$

$\Omega_{e}$ is a domain of volume $\left|\Omega_{e}\right|=M / \rho_{e}$ and $M=\int_{\Omega} \rho_{0}(\xi) d \xi$.

Using inequality (2.12) we can prove the following global existence theorem.

THEOREM 1. Let $f=0, r=0, S \in W_{2}^{5 / 2}, v_{0} \in W_{2}^{2}(\Omega), \rho_{0} \in W_{2}^{2}(\Omega), \theta_{0} \in W_{2}^{2}(\Omega)$, $u_{t}(0) \in W_{2}^{1}(\Omega), \vartheta_{t}(0) \in W_{2}^{1}(\Omega), u_{t t}(0) \in L_{2}(\Omega), \vartheta_{t t}(0) \in L_{2}(\Omega)$ (where $u_{t}(0), u_{t t}(0)$, $\vartheta_{t}(0), \vartheta_{t t}(0)$ are calculated from equations $(2.2)$ and $\left.(2.4)\right)$. Let $\nu, \mu, \varkappa$ be positive constants and $\nu>(1 / 3) \mu ; p \in C^{3}\left(\mathbb{R}^{2}\right) ; p_{\rho}>0, p_{\theta}>0$ for $\rho, \theta>0 ; c_{v} \in C^{2}\left(\mathbb{R}^{2}\right)$ and assume 
that the following compatibility conditions are satisfied:

$$
\begin{aligned}
& \left.\partial_{t}^{i}\left\{\left[\mathbb{D}_{u}(u)-\left(p(\eta, \vartheta)-p_{0}\right)\right] \bar{n}_{u}\right\}\right|_{t=0}=0, \quad i=0,1, \text { on } S, \\
& \left.\partial_{t}^{j}\left[\varkappa \bar{n}_{u} \cdot \nabla_{u} \vartheta-\varkappa_{a}\left(\theta_{a}-\vartheta\right)\right]\right|_{t=0}=0, \quad i=0,1, \quad \text { on } S .
\end{aligned}
$$

Moreover, let the following assumptions be satisfied: $\varphi(0) \leq \varepsilon ; l>0$ is a constant such that $\rho_{e}-l>0, \theta_{0}-l>0$ and

$$
\rho_{1}<\rho_{0}<\rho_{2}, \quad \theta_{1}<\theta_{0}<\theta_{2},
$$

where $\rho_{1}=\rho_{e}-l, \rho_{2}=\rho+l, \theta_{1}=\theta_{a}-l, \theta_{2}=\theta_{e}+l$;

$$
\int_{\Omega} \rho_{0} v_{0} \cdot(a+b \times \xi) d \xi=0
$$

where $a$ and $b$ are arbitrary constant vectors. Then for sufficiently small $\varepsilon$ there exists a unique global solution of $(1.1)-(1.9),\left(1.10^{\prime}\right)$ such that $\left(v, \theta_{\sigma}, \rho_{\sigma}\right) \in \mathfrak{M}(t)$ for $t \in \mathbb{R}_{+}$, $S_{t} \in W_{2}^{5 / 2}$ for $t \in \mathbb{R}_{+}$and

$$
\varphi(t) \leq c \varepsilon \quad \text { fort } \in \mathbb{R}_{+},
$$

where $c>0$ is a constant depending on $\Omega, \rho_{1}, \rho_{2}, \theta_{1}, \theta_{2}, p, c_{v}, \nu, \mu, \varkappa$.

Apart from inequality (2.12) we use in the proof of Theorem 1 the following estimate which holds for the local solution of $(2.2)-(2.5),\left(2.6^{\prime}\right),(2.7)$ :

$$
\|u\|_{\mathcal{A}_{0 T, \Omega}}^{2}+\left\|\vartheta_{\sigma}\right\|_{\mathcal{A}_{0 T, \Omega}}^{2}+\left\|\eta_{\sigma}\right\|_{\mathcal{B}_{0 T, \Omega}}^{2} \leq C_{1}(T) \varphi(0),
$$

where $T$ is the time of local existence; $\mathcal{A}_{0 T, \Omega}, \mathcal{B}_{0 T, \Omega}$ are given by $(2.9)-(2.10)$, and $C_{1}$ is an increasing function of $T$. Inequality (2.13) rewritten in the Eulerian coordinates yields for $t \leq T$

$$
\sup _{0 \leq t^{\prime} \leq t} \varphi\left(t^{\prime}\right)+\int_{0}^{t} \Phi\left(t^{\prime}\right) d t^{\prime} \leq C_{2}(T) \varphi(0),
$$

where $C_{2}$ is an increasing function of $T$.

In the process of extending the solution step by step to all $t>0$, estimate (2.14) implies step by step that $\rho$ and $\theta$ remain in the intervals $\left(\rho_{1}, \rho_{2}\right)$ and $\left(\theta_{1}, \theta_{2}\right)$, respectively, for all $t$.

Inequalities (2.13) and (2.14) allow to extend the solution and to control the shape of $\Omega_{t}$ which will be explained in the next section.

\section{Differences in the ways of controlling the free boundary in two cases: $\sigma=0$ and $\sigma>0$.}

$1^{\circ}$ The case of $\sigma>0$. Consider a free boundary drop problem with $k=0$ and $\sigma>0$. As usual in the case of $\sigma>0$ we assume:

(3.1) $\Omega$ is close to a ball and $S$ is described by the equation: $|\xi|=\widetilde{R}(\omega), \omega \in S^{1}$, where $S^{1}$ is the unit sphere.

Then from the relation (2.1) connecting Lagrangian and Eulerian coordinates it follows that $\Omega_{t}$ is also close to a ball and $S_{t}(t \leq T)$ is described by

$$
|x|=R(\omega, t), \quad \omega \in S^{1},
$$

where $R(\omega, 0)=\widetilde{R}(\omega), T$ is the time of local existence. 
The boundary condition (1.8) can be presented in the form

$$
H+\frac{2}{R_{e}}=\frac{1}{\sigma} \bar{n} \cdot \mathbb{T}\left(v, p_{\sigma}\right) \bar{n} \quad \text { on } S_{t},
$$

where in the incompressible case $R_{e}=R_{0}=\left(\frac{3}{4 \pi}|\Omega|\right)^{1 / 3}$, while in the compressible case $R_{e}$ is given in the definition of an equilibrium solution.

Using (3.2) we can write condition (3.3) in the form

$$
H[R]+\frac{2}{R_{e}}=h(\omega),
$$

where $H[R]$ is the double mean curvature of $S_{t}$ expressed in spherical coordinates, i.e.

$$
H[R]=\frac{1}{R \sin \varphi_{2}}\left(\frac{\partial}{\partial \varphi_{1}} \frac{R_{\varphi_{1}}}{\sin \varphi_{2} \sqrt{R^{2}+|\nabla R|^{2}}}+\frac{\partial}{\partial \varphi_{2}} \frac{\sin \varphi_{2} R_{\varphi_{2}}}{\sqrt{R^{2}+|\nabla R|^{2}}}\right)-\frac{2}{\sqrt{R^{2}+|\nabla R|^{2}}} .
$$

Now, following [Sol1] cover $S^{1}$ by a finite number of domains $S^{\prime}$ having sufficiently small diameters. Take a function $\zeta=\zeta(\varphi)$ such that $\zeta=1$ on $S^{\prime}, \zeta=0$ on $S^{1} \backslash S^{\prime \prime}$, $\bar{S}^{\prime} \subset S^{\prime \prime}$ and $0 \leq \zeta \leq 1$. Next, denote $R_{*}=R-R_{e}, \widetilde{R}_{*}=\zeta R_{*}$. Then, by applying the formula

$$
\frac{1}{R_{e}}-\frac{1}{\sqrt{R^{2}+|\nabla R|^{2}}}=\frac{\left(R-R_{e}\right)\left(R+R_{e}\right)+|\nabla R|^{2}}{R_{e} \sqrt{R^{2}+|\nabla R|^{2}}\left(R_{e}+\sqrt{R^{2}+|\nabla R|^{2}}\right.} \equiv A
$$

equation (3.4) takes the form of the following elliptic equation:

where

$$
\sum_{\gamma, \delta=1}^{2} A_{\gamma \delta}(\varphi) \frac{\partial^{2} \widetilde{R}_{*}}{\partial \varphi_{\gamma} \partial \varphi_{\delta}}+\sum_{\gamma=1}^{2} A_{\gamma}(\varphi) \frac{\partial \widetilde{R}_{*}}{\partial \varphi_{\gamma}}=G
$$

$$
G \equiv 2 \sum_{\gamma, \delta=1}^{2} A_{\gamma \delta}(\varphi) \frac{\partial \zeta}{\partial \varphi_{\gamma}} \frac{\partial R_{*}}{\partial \varphi_{\delta}}+R_{*} \sum_{\gamma, \delta=1}^{2} A_{\gamma \delta}(\varphi) \frac{\partial^{2} \zeta}{\partial \varphi_{\gamma} \partial \varphi_{\delta}}+\sum_{\gamma=1}^{2} A_{\gamma}(\varphi) \frac{\partial \zeta}{\partial \varphi_{\gamma}}-2 A \zeta+h \zeta .
$$

Spherical coordinates have been chosen so that $\sin \varphi_{1} \geq c_{0}>0$. Therefore $A_{\gamma \delta}, A_{\gamma}$, $A \in W_{2}^{1 / 2+l}\left(S^{1}\right)$, and these coefficients do not depend on $R_{\varphi \varphi}, \varphi=\left(\varphi_{1}, \varphi_{2}\right)$.

In order to control the free boundary in this case (and as a consequnece to extend the solution for all $t$ ) we use the regularity properties of the above elliptic equation. More precisely, we use the following theorem.

Theorem 2 (see [Sol1, Z2]). Let $R \in W_{2}^{3 / 2+l}\left(S^{1}\right), l \in(1 / 2,1)$ be a solution of equation (3.4) satisfying inequality

$$
\sup _{S^{1}}\left|R(\omega, t)-R_{e}\right|+\sup _{S^{1}}|\nabla R(\omega, t)| \leq \widehat{\delta} R_{e}
$$

with sufficiently small $\widehat{\delta}$. If $h \in W_{2}^{s}\left(S^{1}\right), s \in[0,1]$, then

$$
\left\|R-R_{e}\right\|_{W_{2}^{2+s}\left(S^{1}\right)} \leq c_{1}\|h\|_{W_{2}^{s}\left(S^{1}\right)}+c_{2}\left\|R-R_{e}\right\|_{L_{2}\left(S^{1}\right)},
$$

where $c_{1}, c_{2}$ are constants and $c_{2}$ can depend on $\|R\|_{W_{2}^{l+3 / 2}\left(S^{1}\right)}$. Moreover, if $R \in W_{2}^{2+s}\left(S^{1}\right)$ and $h \in W_{2}^{1+s}\left(S^{1}\right), s \in(0, \infty)$, then

$$
\left\|R-R_{e}\right\|_{W_{2}^{3+s}\left(S^{1}\right)} \leq c_{3}\|h\|_{W_{2}^{1+s}\left(S^{1}\right)}+c_{4}\left\|R-R_{e}\right\|_{L_{2}\left(S^{1}\right)},
$$

where $c_{3}, c_{4}$ are constants and $c_{4}$ can depend on $\|R\|_{W_{2}^{2+s}\left(S^{1}\right)}$. 
Theorem 2 is essential to all proofs of global existence and stability theorems for drop problems. The norm $\left\|R-R_{e}\right\|_{L_{2}\left(S^{1}\right)}$ is estimated in each case by using conservation laws of: energy, momentum and mass, and in order to estimate the norms $\|h\|_{W_{2}^{s}\left(S^{1}\right)}$ or $\|h\|_{W_{2}^{1+s}\left(S^{1}\right)}$ we need estimates of $v$ and $p_{\sigma}$. Such estimates are derived in different ways in dependence on what motion we consider. Thus, if we assume that $S \in W_{2}^{3+s}$ for some $s>0$, we obtain by Theorem 2 the same regularity of the boundary for all $t>0$, i.e. $S_{t} \in W_{2}^{3+s}$. Moreover, for data sufficiently close to an equilibrium state we can usually prove that the free boundary $S_{t}$ remains close to a ball of radius $R_{e}$ for all $t$.

For surface waves problems, a theorem analogous to Theorem 2 can be proved for function $F\left(x^{\prime}, t\right)$ describing a free boundary in this case (see [TTan]).

$2^{\circ}$ The case of $\sigma=0$. Now, we consider a drop problem with $\sigma=0$. Then the LaplaceBeltrami operator $\Delta_{S_{t}}(t)$ does not occur in boundary condition (1.8). Therefore, we cannot apply Theorem 2 and the way of controlling a free boundary is different than for $\sigma>0$. In this case we prove the following differential inequality

$$
\frac{d \bar{\varphi}}{d t}+c_{1} \Phi \leq 0 \quad \text { for } t \leq T,
$$

where $T$ is the time of local existence; $c_{1}>0$ is a constant.

In the case of a compressible heat-conducting fluid, $\bar{\varphi}=\bar{\varphi}(t)$ is a function equivalent to

$$
\varphi(t)=\sum_{i \in N_{1}}\left(\left\|\partial_{t}^{i} v(t)\right\|_{X_{i}\left(\Omega_{t}\right)}^{2}+\left\|\partial_{t}^{i} \theta_{\sigma}(t)\right\|_{X_{i}\left(\Omega_{t}\right)}^{2}+\left\|\partial_{t}^{i} \rho_{\sigma}(t)\right\|_{X_{i}\left(\Omega_{t}\right)}^{2}\right),
$$

where $N_{1}$ is a subset of $\mathbb{N} \cup\{0\}$, and $X_{i}\left(\Omega_{t}\right), i \in N_{1}$, is a certain function space, usually of the Sobolev type; $\theta_{\sigma}=\theta-\theta_{e}, \rho_{\sigma}=\rho-\rho_{e} ;(v, \theta, \rho)$ is the local solution of problem $(1.1)-(1.9),\left(1.10^{\prime}\right) ; \theta_{e}$ and $\rho_{e}$ are the constants defined in Definition 2. Moreover,

$$
\Phi(t)=\sum_{j \in N_{2}}\left(\left\|\partial_{t}^{j} v(t)\right\|_{Y_{j}\left(\Omega_{t}\right)}^{2}+\left\|\partial_{t}^{j} \theta_{\sigma}(t)\right\|_{Y_{j}\left(\Omega_{t}\right)}^{2}\right)+\sum_{k \in N_{3}}\left\|\partial_{t}^{k} \partial_{t}^{j} \rho_{\sigma}(t)\right\|_{Z_{k}\left(\Omega_{t}\right)}^{2},
$$

where $Y_{j}\left(\Omega_{t}\right), j \in N_{2}$ and $Z_{k}\left(\Omega_{t}\right), k \in N_{3}\left(N_{2}, N_{3}\right.$ are subsets of $\left.\mathbb{N} \cup\{0\}\right)$ are spaces such that

$$
\Phi \geq c_{2} \varphi
$$

In the compressible barotropic case we omit in formulas (3.8)-(3.9) the terms $\sum_{i \in N_{1}}\left\|\partial_{t}^{i} \theta_{\sigma}(t)\right\|_{X_{i}\left(\Omega_{t}\right)}^{2}$ and $\sum_{j \in N_{2}}\left\|\partial_{t}^{j} \theta_{\sigma}(t)\right\|_{Y_{j}\left(\Omega_{t}\right)}^{2}$, respectively.

Finally, for an incompressible fluid $\bar{\varphi}$ is equivalent to $\varphi(t)=\sum_{i \in N_{1}}\left\|\partial_{t}^{i} v(t)\right\|_{X_{i}\left(\Omega_{t}\right)}^{2}$ and $\Phi(t)=\sum_{j \in N_{2}}\left\|\partial_{t}^{j} v(t)\right\|_{Y_{j}\left(\Omega_{t}\right)}^{2}$, where $v$ is the local solution of (1.19)-(1.20), (1.4)-(1.5), (1.8)-(1.9).

Thus, we have

$$
c_{3} \varphi(t) \leq \bar{\varphi}(t) \leq c_{4} \varphi(t) \quad \text { for } t \leq T,
$$

where in the general heat-conducting case constants $c_{3}, c_{4}>0$ depend on $\rho_{1}, \rho_{2}, \theta_{1}, \theta_{2}$, $\mu, \nu, \varkappa, c_{v}, p,\left\|\int_{0}^{t} v d t^{\prime}\right\|_{W_{2}^{3}\left(\Omega_{t}\right)}$ and $\rho_{1}, \rho_{2}, \theta_{1}, \theta_{2}$ are positive constants such that

$$
\rho_{1}<\rho(x, t)<\rho_{2}, \quad \theta_{1}<\theta(x, t)<\theta_{2} \quad \text { for } x \in \bar{\Omega}_{t}, t \in[0, T] .
$$


The constant $c_{1}$ in (3.7) depends on the same quantities as $c_{3}$ and $c_{4}$. It depends also on $\left\|S_{t}\right\|_{W_{2}^{5 / 2}}$ and the constants from the imbedding lemmas and the Korn inequalities which depend on $\Omega_{t}, t \leq T$.

Inequalities (3.7), (3.10), (3.11) imply

$$
\frac{d \bar{\varphi}}{d t}+c_{5} \bar{\varphi} \leq 0 \quad \text { for } t \leq T
$$

Hence

$$
\bar{\varphi}(t) \leq \bar{\varphi}(0) e^{-c_{5} t} \quad \text { for } t \leq T
$$

and

$$
\varphi(t) \leq \frac{c_{4}}{c_{3}} \varphi(0) e^{-c_{5} t} \quad \text { for } t \leq T
$$

Moreover

$$
\bar{\varphi}(t)+c_{1} \int_{0}^{t} \Phi\left(t^{\prime}\right) d t^{\prime} \leq \bar{\varphi}(0) \quad \text { for } t \leq T .
$$

In the incompressible case it suffices to take $N_{1}=\{0\}, N_{2}=\{0\}$ and $X_{0}\left(\Omega_{t}\right)=$ $L_{2}\left(\Omega_{t}\right), Y_{0}\left(\Omega_{t}\right)=W_{2}^{1}\left(\Omega_{t}\right)$ (see [Sol2]). Then $\bar{\varphi}(t)=\varphi(t)=\|v(t)\|_{L_{2}\left(\Omega_{t}\right)}^{2}$ and inequalities (3.7) and (3.13) follow from the energy conservation law and the Korn inequality. However, the solvability of problem $(1.19)-(1.20),(1.4)-(1.5),(1.8)-(1.9)$ is proved in [Sol2] in spaces of more regular functions $v$ than $L_{2}\left(\Omega_{t}\right)$.

In the compressible case, the functions $\varphi(t)$ and $\Phi(t)$ with the lowest possible regularity of functions $v, \theta_{\sigma}, \rho_{\sigma}$ are defined as follows

$$
\begin{aligned}
\varphi(t)= & \sum_{i=0}^{2}\left(\left\|\partial_{t}^{i} v(t)\right\|_{W_{2}^{2-i}\left(\Omega_{t}\right)}^{2}+\left\|\partial_{t}^{i} \theta_{\sigma}(t)\right\|_{W_{2}^{2-i}\left(\Omega_{t}\right)}^{2}+\left\|\partial_{t}^{i} \rho_{\sigma}(t)\right\|_{W_{2}^{2-i}\left(\Omega_{t}\right)}^{2}\right) \\
\Phi(t)= & \sum_{j=0}^{2}\left(\left\|\partial_{t}^{j} v(t)\right\|_{W_{2}^{3-j}\left(\Omega_{t}\right)}^{2}+\left\|\partial_{t}^{j} \theta_{\sigma}(t)\right\|_{W_{2}^{3-j}\left(\Omega_{t}\right)}^{2}\right)+\left\|\rho_{\sigma}(t)\right\|_{W_{2}^{2}\left(\Omega_{t}\right)}^{2} \\
& +\left\|\partial_{t} \rho_{\sigma}(t)\right\|_{W_{2}^{2}\left(\Omega_{t}\right)}^{2}+\left\|\partial_{t}^{2} \rho_{\sigma}(t)\right\|_{W_{2}^{1}\left(\Omega_{t}\right)}^{2} .
\end{aligned}
$$

In this case inequality (3.7) follows from (2.12) if we assume that $\varphi(0) \leq \varepsilon$ with $\varepsilon$ sufficiently small. In fact, then by $(2.14)$ we get

$$
\sup _{0 \leq t \leq T} \varphi(t) \leq C_{2}(T) \varepsilon
$$

and by $(2.13)$

$$
\begin{aligned}
\left\|\int_{0}^{t} v d t^{\prime}\right\|_{W_{2}^{3}\left(\Omega_{t}\right)} & \leq c_{6}\left\|\int_{0}^{t} u d t^{\prime}\right\|_{W_{2}^{3}(\Omega)} \leq c_{6} T^{1 / 2}\|u\|_{A_{T, \Omega}} \\
& \leq c_{6} C_{1}(T) T^{1 / 2} \varepsilon^{1 / 2} \leq c_{6} \bar{\varepsilon} \quad \text { for } t \leq T
\end{aligned}
$$

if $\varepsilon$ is sufficiently small.

Therefore, for $\varepsilon, \bar{\varepsilon}$ sufficiently small estimates (3.19)-(3.20) and inequality (2.12) yield (3.7). 
Moreover, since by (3.20)

$$
|x-\xi|=\left|\int_{0}^{t} u d t^{\prime}\right| \leq c_{7}\left\|\int_{0}^{t} u d t^{\prime}\right\|_{W_{2}^{3}(\Omega)} \leq c_{7} C_{1}(T) T^{1 / 2} \varepsilon^{1 / 2} \leq c_{7} \bar{\varepsilon} \quad \text { for } t \leq T,
$$

both the volume and the shape of $\Omega_{t}(t \leq T)$ do not change much if $\bar{\varepsilon}$ is sufficiently small.

Next, assuming that the initial conditions $\rho_{0}, \theta_{0}$ and the equilibrium solution $\left(\rho_{e}, \theta_{e}\right)$ satisfy inequalities (3.12) we prove by using estimate (2.13) that inequalities (3.12) are satisfied for $x \in \bar{\Omega}_{t}, t \in[0, T]$.

Therefore, estimate (3.21) and inequalities (3.12) for $x \in \bar{\Omega}_{t}, t \in[0, T]$ imply that if we assume (3.11) for $\varphi(0)$ and $\bar{\varphi}(0)$ with $c_{3}, c_{4}$ depending on $\rho_{1}, \rho_{2}, \theta_{1}, \theta_{2}, \mu, \nu, c_{v}, p$, $\varkappa$, we obtain this estimate satisfied by $\varphi(t)$ and $\bar{\varphi}(t)$ for $t \leq T$ with the same constants $c_{3}, c_{4}$.

Hence, by (3.14) we get (3.15). As a consequence, we have

$$
\varphi(t) \leq \frac{c_{4}}{c_{3}} \varepsilon \quad \text { for } t \leq T .
$$

Therefore, for sufficiently small $\varepsilon$ the solution can be extended to the interval $[T, 2 T]$. This solution satisfies in $[T, 2 T]$ the inequality

$$
\left\|u_{T}\right\|_{\mathcal{A}_{T, \Omega_{T}}}^{2}+\left\|\vartheta_{T \sigma}\right\|_{\mathcal{A}_{T, \Omega_{T}}}^{2}+\left\|\eta_{T \sigma}\right\|_{\mathcal{B}_{T, \Omega_{T}}}^{2} \leq C_{1}(T) \varphi(T) .
$$

where $\left(u_{T}, \vartheta_{T} \sigma, \eta_{T} \sigma\right)$ denotes $\left(v, \theta_{\sigma}, \rho_{\sigma}\right)$ written in the Lagrangian coordinates $\xi_{T} \in \Omega_{T}$, i.e. $\xi_{T}=\xi+\int_{0}^{T} u\left(\xi, t^{\prime}\right) d t^{\prime}$.

Moreover, using (3.16), (3.22), (3.23), (3.11) we get for $t \leq 2 T$

$$
\begin{aligned}
\left\|\int_{0}^{t} u d t^{\prime}\right\|_{W_{2}^{3}(\Omega)} & \leq\left\|\int_{0}^{T} u d t^{\prime}\right\|_{W_{2}^{3}(\Omega)}+\left\|\int_{T}^{t} u d t^{\prime}\right\|_{W_{2}^{3}(\Omega)} \\
& \leq c_{8} T^{1 / 2}\left[\left(c_{1} \int_{0}^{T} \Phi\left(t^{\prime}\right) d t^{\prime}\right)^{1 / 2}+\left\|u_{T}\right\|_{\mathcal{A}_{T, \Omega_{T}}}\right] \\
& \leq c_{8} c_{4}^{1 / 2} T^{1 / 2} \varepsilon^{1 / 2}\left[1+\left(C_{1}(T) / c_{3}\right)^{1 / 2}\right] \leq \bar{\varepsilon}
\end{aligned}
$$

and

$$
\begin{aligned}
|x-\xi| & =\left|\int_{0}^{t} u d t^{\prime}\right| \leq c_{7}\left\|\int_{0}^{t} u d t^{\prime}\right\|_{W_{2}^{3}(\Omega)} \\
& \leq c_{7} c_{8} c_{4}^{1 / 2} T^{1 / 2} \varepsilon^{1 / 2}\left[1+\left(C_{1}(T) / c_{3}\right)^{1 / 2}\right] \leq c_{7} \bar{\varepsilon}
\end{aligned}
$$

if $\varepsilon$ is sufficiently small.

In view of $(3.25)$ the volume and the shape of $\Omega_{t}$ changes in $[0,2 T]$ no more than they do in $[0, T]$.

Inequality (3.24) implies

$$
\left\|\int_{0}^{t} v d t^{\prime}\right\|_{W_{2}^{3}\left(\Omega_{t}\right)} \leq c_{6}\left\|\int_{0}^{t} u d t^{\prime}\right\|_{W_{2}^{3}(\Omega)} \leq c_{6} \bar{\varepsilon} \quad \text { for } t \leq 2 T .
$$

Using (3.23) and (3.15) we also obtain

$$
\sup _{0 \leq t \leq 2 T} \varphi(t) \leq \max \left(\sup _{0 \leq t \leq T} \varphi(t), \sup _{T \leq t \leq 2 T} \varphi(t)\right) \text { leq } \max \left(\frac{c_{4}}{c_{3}} \varepsilon, C_{2}(T) \frac{c_{4}}{c_{3}} \varepsilon\right) .
$$


Now, since the solution exists in the interval $[0,2 T]$ and in view of (3.25) we can derive inequality (2.12) for $t \leq 2 T$. Therefore, estimates (3.26)-(3.27) with sufficiently small $\bar{\varepsilon}$ and $\varepsilon$ imply differential inequality (3.7) for $t \leq 2 T$.

Hence (3.13)-(3.15) also hold for $t \leq 2 T$ and

$$
\bar{\varphi}(t)+c_{1} \int_{T}^{t} \Phi\left(t^{\prime}\right) d t^{\prime} \leq \bar{\varphi}(T) \quad \text { for } T \leq t \leq 2 T .
$$

This way the solution can be extended to the interval $[2 T, 3 T]$.

Now, assume that there exists a solution in $[0, l T], l \geq 3$, satisfying:

$$
\begin{gathered}
\left\|u_{j T}\right\|_{\mathcal{A}_{j T, \Omega_{j} T}}^{2}+\left\|\vartheta_{j T \sigma}\right\|_{\mathcal{A}_{j T, \Omega_{j}}}^{2}+\left\|\eta_{j T \sigma}\right\|_{\mathcal{B}_{j T, \Omega_{j} T}}^{2} \leq C_{1}(T) \varphi(j T), \quad j=0, \ldots, l-1, \\
\bar{\varphi}(t) \leq \bar{\varphi}(0) e^{-c_{5} t} \quad \text { for } t \leq(l-1) T, \\
\varphi(t) \leq \frac{c_{4}}{c_{3}} \varphi(0) e^{-c_{5} t} \quad \text { for } t \leq(l-1) T, \\
\bar{\varphi}(t)+c_{1} \int_{j T}^{t} \Phi\left(t^{\prime}\right) d t^{\prime} \leq \bar{\varphi}(j T) \quad \text { for } j T \leq t \leq(j+1) T, j=0, \ldots, l-2,
\end{gathered}
$$

where $u_{j T}, \vartheta_{j T \sigma}, \eta_{j T \sigma}$ denote $v, \theta_{\sigma}, \rho_{\sigma}$ written in the Lagrangian coordinates $\xi_{j T} \in \Omega_{j T}$.

Assume also that the volume and the shape of $\Omega_{t}$ change in $[0,(l-1) T]$ no more than they do in $[0, T]$ and that

$$
\left\|\int_{0}^{t} u d t^{\prime}\right\|_{W_{2}^{3}(\Omega)} \leq \bar{\varepsilon} \quad \text { for } t \leq(l-1) T
$$

with sufficiently small $\bar{\varepsilon}$.

Hence, assuming that $\bar{\varepsilon}$ is sufficiently small we obtain for $0 \leq t \leq l T$

$$
\begin{aligned}
|x-\xi| & \left.=\left|\int_{0}^{t} u\left(\xi, t^{\prime}\right) d t^{\prime}\right| \leq c_{7}\left\|\int_{0}^{t} u d t^{\prime}\right\|_{W_{2}^{3}(\Omega)}+\left\|\int_{(l-1) T}^{t} u d t^{\prime}\right\|_{W_{2}^{3}(\Omega)}\right) \\
& \leq c_{7}\left(\sum_{j=0}^{l-2}\left\|\int_{j T}^{(j+1) T} u d t^{\prime}\right\|_{W_{2}^{3}(\Omega)}+\left\|u_{(l-1) T}\right\|_{A_{T, \Omega_{(l-1) T}}}\right] \\
& \leq c_{7} c_{8} T^{1 / 2}\left[\sum_{j=0}^{l-2}\left(\int_{j T}^{(j+1) T} c_{1} \Phi\left(t^{\prime}\right) d t^{\prime}\right)^{1 / 2}+\|{ }^{1 / 2}\right] \\
& \left.\leq c_{7} c_{8} T^{1 / 2}\left[\sum_{j=0}^{l-2}(\bar{\varphi}(j T))^{1 / 2}+C_{1}(T)\left(\frac{c_{4}}{c_{3}} \varepsilon\right)^{1 / 2}\right)^{1 / 2}\right\} \\
& \leq c_{7} c_{8} T^{1 / 2}\left\{\left[\bar{\varphi}(0)\left(1+e^{-c_{5} T}+e^{-2 c_{5} T}+\ldots\right)\right]_{1}(T)\left(\frac{c_{4}}{c_{3}} \varepsilon\right)^{1 / 2}\right\} \\
& \leq c_{7} c_{8} c_{4}^{1 / 2} T^{1 / 2} \varepsilon^{1 / 2}\left[\frac{1}{\left(1-e^{-c_{5} T}\right)^{1 / 2}}+\left(\frac{C_{1}(T)}{c_{3}}\right)^{1 / 2}\right]
\end{aligned}
$$

if $\varepsilon$ is sufficiently small in dependence on $\bar{\varepsilon}$.

Thus, the volume and the shape of $\Omega_{t}$ change in $[0, l T]$ no more than they do in $[0,(l-1) T]$. These changes of the volume and the shape are as small as we want if we assume that $\bar{\varepsilon}$ is sufficiently small. 
Therefore, we can control the free boundary of $\Omega_{t}$ in the case of $\sigma=0$. At the same time, this way, we can extend the solution to a global one.

The difficulties connected with controlling the free boundary in the surface waves problems without surface tension are discussed by Beale [B] for incompressible fluids and Ströhmer [St] for compressible ones.

\section{References}

[A] H. Abels, Stokes equations in asymptotically flat domains and the motion of a free surface, D 17 (Diss. TU Darmstadt), Shaker Verlag, Aachen 2003.

[B] T. Beale, The initial value problem for the Navier-Stokes equation with a free surface, Comm. Pure Appl. Math. 34 (1981), 359-392.

[JinPad] B. J. Jin and M. Padula, On existence of compressible viscous flow in a horizintal layer with free upper surfaces, Università degli Studi di Ferrara, Dipartimento di Matematica, Preprint n. 317, 2002.

[LanLif] L. Landau and E. Lifschitz, Hydrodynamics, Nauka, Moscow 1986 (in Russian); English transl.: Fluid Mechanics, Pergamon Press, Oxford, 1987.

[MZaj] P. B. Mucha and W. M. Zajączkowski, On local existence of solutions of the free boundary problem for an incompressible viscous self-gravitating fluid motion, Appl. Math. 27 (2000), 319-333.

[Ser] J. Serrin, Mathematical Principles of Classical Fluid Mechanics, Handbuch der Physik. VIII/1, Springer, Berlin-Heidelberg-New York, 1959, 125-263.

[Sol1] V. A. Solonnikov, On an unsteady flow of a finite mass of a liquid bounded by a free surface, Zap. Nauchn. Sem. LOMI 152 (1986), 137-157 (in Russian); English transl.: J. Soviet Math. 40 (1988), 672-686.

[Sol2] V. A. Solonnikov, On an unsteady motion of an isolated volume of a viscous incompressible fluid, Izv. Akad. Nauk SSSR Ser. Mat. 51 (1987), 1065-1087; English transl.: Math. USSR-Izv. 31 (1988), 381-405.

[Sol3] V. A. Solonnikov, On nonstationary motion of a finite isolated mass of self-gravitating fluid, Algebra and Analysis 1 (1989), 207-249; English transl.: Leningrad Math. J. 1 (1990), 227-276.

[Sol4] V. A. Solonnikov, Solvability of the problem of evolution of a viscous incompressible fluid bounded by a free surface on a finite time interval, Algebra and Analysis 3 (1991), 222-257; English transl.: St. Petersburg Math. J. 3 (1992), 189-220.

[SolT1] V. A. Solonnikov and A. Tani, Free boundary problem for a viscous compressible flow with the surface tension, in: Constantine Caratheodory: An International Tribute, T. M. Rassias (ed.), 2 (1991), 1270-1303.

[SolT2] V. A. Solonnikov and A. Tani, Evolution free boundary problem for equations of motion of viscous compressible barotropic liquid, Lecture Notes in Math. 1530, Springer, 1992, 30-55.

[St] G. Ströhmer, About the decay of surface waves on viscous fluids without surface tension, in: Evolution Equations, Banach Center Publ. 60, Warszawa, 2003, 55-72.

[StZaj] G. Ströhmer and W. M. Zajączkowski, Local existence of solutions of the free boundary problem for the equations of compressible barotropic viscous self-gravitating fluids, Appl. Math. 26 (1999), 1-31. 
[Syl] D. Sylvester, Large time existence of small viscous surface waves without surface tension, Comm. Partial Diff. Equations 15 (1990), 823-903.

[TanT] N. Tanaka and A. Tani, Surface waves for a compressible viscous fluid, J. Math. Fluid Mech. 5 (2003), 303-363.

[T] A. Tani, Small-time existence for the three-dimensional incompressible Navier-Stokes equations with a free surface, Arch. Rational Mech. Anal. 133 (1996), 299-331.

[TTan] A. Tani and N. Tanaka, Large-time existence of surface waves in incompressible viscous fluids with or without surface tension, Arch. Rational Mech. Anal. 130 (1995), 303-314.

[Z1] E. Zadrzyńska, Free boundary problem for a viscous heat-conducting flow with surface tension, Topol. Methods Nonlin. Anal. 19 (2002), 313-338.

[Z2] E. Zadrzyńska, Free boundary problems for nonstaionary Navier-Stokes equations, Dissertationes Math. 424, 2004.

[ZZaj1] E. Zadrzyńska and W. M. Zajączkowski, Local existence of solutions of a free boundary problem for equations of compressible viscous heat-conducting fluids, Appl. Math. 25 (1998), 179-220.

[ZZaj2] E. Zadrzyńska and W. M. Zajączkowski, On nonstationary motion of a fixed mass of a viscous compressible barotropic fluid bounded by a free boundary, Colloq.Math. 79 (1999), 283-310.

[ZZaj3] E. Zadrzyńska and W. M. Zajączkowski, Differential inequlities for general fluid motions bounded by a free surface, J. Appl. Anal., to appear.

[ZZaj4] E. Zadrzyńska and W. M. Zajączkowski, On nonstationary motion of a fixed mass of a general fluid bounded by a free surface, in: Banach Center Publications 60 (2003), 253-266.

[Zaj] W. M. Zajączkowski, On nonstationary motion of a compressible barotropic viscous capillary fluid bounded by a free surface, SIAM J. Math. Anal. 25 (1994), 1-84. 